(2) OPEN ACCESS
- Additional material is published online only. To view, please visit the journal online (http://dx.doi.org/10.1136/ thoraxjnl-2020-215520).

${ }^{1}$ Servei de Pneumologia, Grup de Recerca Pneumològic, Institut d'Investigacions Biomèdiques de Bellvitge (IDIBELL), Hospital Universitari de Bellvitge, Hospital de Llobregat, Barcelona, Spain ${ }^{2}$ Instituto Nacional de Enfermedades Respiratorias Ismael Cosio Villegas, Mexico City, Mexico

${ }^{3}$ Facultad de Ciencias, Universidad Nacional Autonoma de Mexico, Mexico City, Mexico

\section{Correspondence to}

Dr Leslie Chavez-Galan, Laboratory of Integrative Immunology, Instituto Nacional de Enfermedades Respiratorias, 14080 Mexico, Mexico: Ichavez_galan@iner.gob.mx

CM and IB-R contributed equally.

Received 11 June 2020 Revised 21 September 2020 Accepted 24 September 2020 Published Online First 9 December 2020

Check for updates

(c) Author(s) (or their employer(s)) 2021. Re-use permitted under CC BY-NC. No commercial re-use. See rights and permissions. Published by BMJ.

To cite: Machahua $C$, Buendia-Roldan I, OcañaGuzman R, et al. Thorax 2021:76:152-160

\title{
CD4+T cells in ageing-associated interstitial lung abnormalities show evidence of pro-inflammatory phenotypic and functional profile
}

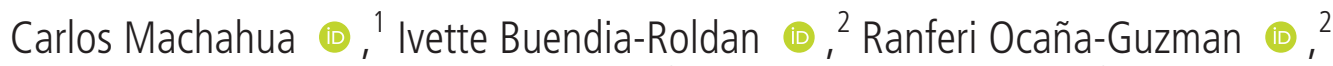 \\ María Molina-Molina, ${ }^{1}$ Annie Pardo (ㄷ), ${ }^{3}$ Leslie Chavez-Galan 지, ${ }^{2}$ \\ Moises Selman ${ }^{2}{ }^{2}$
}

\section{ABSTRACT \\ Background Interstitial lung abnormalities (ILA)} occur in around $10 \%$ of subjects over 60 years, and are associated with a higher rate of all-cause mortality. The pathogenic mechanisms are unclear, and the putative contribution of alterations in the immune response has not been explored. Normal ageing is associated with immune deficiencies, including Naïve T-cell decrease and greater expression of the proliferative-limiting, co-inhibitory receptor killer-cell lectin-like receptor G1 (KLRG1).

Objective To evaluate the frequency and activation state of different T-cell subpopulations in ILA subjects. Methods Peripheral blood mononuclear cells were obtained from 15 individuals with ILA, 21 age-matched controls and 28 healthy young subjects. T-cells phenotype was characterised by flow cytometry, and proliferation and activation by stimulation with anti-CD3/anti-CD28 or phorbol myristate acetate/ionomycin; KLRG1 isoforms were evaluated by western blot and cytokines were quantified by ELISA and Multiplex.

Results A significant increase of Naïve CD4+T cells together with a decrease of central and effector memory CD4+T cells was observed in ILA compared with age-matched controls. CD4+T cells from ILA subjects exhibited greater basal proliferation, which raised after anti-CD3/anti-CD28 stimulation. Additionally, a significant increase in the levels of interleukin- 6 and interferon gamma was observed in isolated CD4+T cells and plasma of ILA subjects. They also displayed fewer KLRG1+/CD4+T cells with an increase of circulating E-cadherin, the ligand of KLRG1+. No changes were observed with CD8+T cell subsets.

Conclusion CD4+T cells from ILA subjects are highly proliferative and show an excessive functional activity, likely related to the loss of KLRG1 expression, which may contribute to an inflammatory state and the development of ILA.

\section{INTRODUCTION}

Lung ageing involves a wide range of structural changes that result in progressive loss of respiratory function. ${ }^{12}$ In addition, cellular changes associated with ageing have been related to the development of some chronic degenerative lung diseases. ${ }^{3}$

Studies have shown that 8 to 10 per cent of adults over 60 years develop interstitial lung

\section{Key messages}

What is the key question?

- Is there a role of abnormalities in the immune system that may contribute to the development of ageing-associated interstitial lung abnormalities (ILA)?

What is the bottom line?

- This study reveals that subjects with ILA showed several alterations in the cellular immune response, including an increased frequency of Naïve CD4+T cells with a decrease of the inhibitory KLRG1 expression resulting in the presence of highly proliferative and proinflammatory CD4+T cells.

Why read on?

- This is the first study that demonstrates a specific association between ILA and phenotypic and functional abnormalities of the immune system.

abnormalities (ILA), defined as several specific patterns of increased lung density observed on chest high-resolution CT (HRCT) scans. ${ }^{4}$ These abnormalities may include ground-glass opacities, reticular abnormalities, diffuse centrilobular nodules, traction bronchiectasis or architectural distortion involving at least $5 \%$ of non-dependent portions of the lung. ${ }^{4-6}$ A growing body of evidence suggests that ILA is associated with functional lung decline, increased respiratory symptoms and increased risk to respiratory-specific mortality. ${ }^{7-9}$ At present, however, the pathogenic mechanisms and the role of the immune system in the development and progression of ILA are unclear.

Ageing is associated with a variety of modifications in the immune system. T cells can be broadly classified into helper T cells (CD4+T) and cytotoxic T cells (CD8+T). CD45 and C-C chemokine receptor 7 (CCR7) are helpful to identify the maturation status of these T cells: Naïve (CCR7+/CD45RA+), central memory ( $\left.\mathrm{T}_{\mathrm{CM}}, \mathrm{CCR} 7+/ \mathrm{CD} 45 \mathrm{RA}-\right)$, effector memory ( $\mathrm{T}_{\mathrm{EM}}$, CCR7-/CD45RA-) and effector memory RA (TEMRA, CCR7-/CD45RA+). ${ }^{10}$

During ageing, the accumulation of differentiated and less proliferative $T$ cells ( $\mathrm{T}_{\mathrm{EM}}$ and TEMRA) and 
the decrease of Naïve T cells represent features of the immune system senescence (immunosenescence). ${ }^{11}$ High-differentiated $\mathrm{T}$ cells have decreased expression of co-stimulator molecules such as CD27 and CD28, and consequently, their proliferation and survival capabilities are affected. ${ }^{12}$ Importantly, the expression of the proliferative-limiting, co-inhibitory receptor killer-cell lectin like receptor G1 (KLRG1) on T cell surface rises with age. KLRG1-transgenic mice have demonstrated that antigen-stimulated T cells in the presence of KLRG1's ligand, E-cadherin, inhibits the proliferative capacity of $\mathrm{CD} 8+\mathrm{T}$ cells, and similarly, KLRG1-expressing CD4+T cells have affected their proliferation capacity. ${ }^{13}{ }^{14}$ KLRG1 could be expressed as a monomer (M), dimer (D), trimer $(\mathrm{T})$ and tetramer $(\mathrm{Tt})$, and has been suggested that the multimerisation of KLRG1 increases the avidity to E-cadherin. ${ }^{15}$

The progressive loss of the immune protection associated with ageing might be crucial in the emergence of respiratory disorders in older populations. Currently, there are no studies approaching specific alterations in the immune system that could contribute to the development of ILA. We hypothesised that T cells from ILA persons have a different phenotype and modified functions compared with an age-matched normal population. In this context, the present study aimed to identify the phenotype of $\mathrm{T}$ cells in individuals with ILA and evaluate their activation/proliferation activities that may participate in the development of ILA.

\section{METHODS}

Detailed methods are provided in the online supplemental file 1.

\section{Study populations}

Respiratory asymptomatic volunteers aged 60 or older have been invited to participate in our 'Lung Ageing Programme'. From this cohort, 15 individuals with ILA and 21 age-matched controls were evaluated. For comparison, we also included 28 healthy young subjects. We obtained demographic and pulmonary function tests data. Two expert thoracic radiologists examined CT scans, and ILA was diagnosed by the existence of different structural alterations including the presence of ground-glass opacities, reticular abnormalities, diffuse centrilobular nodules, honeycombing, traction bronchiectasis, non-emphysematous cysts or architectural distortion involving at least 5\% of non-dependent portions of the lung. CT findings were classified according to the recent classification reported by Fleischner Society. ${ }^{16}$

\section{Preparation of cells}

Peripheral blood mononuclear cells (PBMCs) were collected via ficoll density gradient (Lymphoprep Axis-Shield, Oslo, Norway). The enrichment of total T cells and CD4+T cells was performed by negative selection (Miltenyi Biotec, Bergisch Gladbach, Germany) as described in the online supplemental file 1.

\section{Western blot}

CD4+ and CD4 - T cells were lysed, and total proteins were subjected to SDS-PAGE under non-reducing conditions. Protein bands were detected by incubation with enhanced chemiluminescence reagent (Thermo Scientific, Pierce Biotechnology, Rockford, Illinois), and visualised with the Imaging System from Bio-Rad (ChemiDoc XRS+System) as described in the online supplemental file 1. More information of the used antibodies is indicated in online supplemental figure S1.

\section{Multiparametric cytometry analysis}

PBMCs were obtained and prepared to flow cytometry analysis, as described in the online supplemental file 1 and online supplemental figure S2. More information of the used antibodies is indicated in online supplemental figure S1.

\section{Polyclonal stimulation of $\mathrm{T}$ cells}

Total T cells enrichment were stimulated with polyclonal stimuli. Supernatants were collected for cytokine measurements and cells for proliferation assay, as described in the online supplemental file 1 and online supplemental figure S3. More information of the used antibodies is indicated in online supplemental figure S1.

\section{ELISA determinations in culture supernatants and plasma samples}

Standard ELISA for the cytokine interferon gamma (IFN- $\gamma$ ), interleukin (IL)-2, IL-6 and IL-10 (Biolegend), and for the proteins E-cadherin and granzyme B (R\&D Systems) was performed as described in the online supplemental file 1.

\section{Multiple cytokine assay in supernatant samples}

A Bio-Plex Pro Human cytokine 48-Plex Screening Panel was used as described in the online supplemental file 1 .

\section{Statistical analysis}

Normal distribution was corroborated by the KolmogorovSmirnov test, and data are reported as mean \pm SD or frequency and percentage according to the type of variable. Clinical data were statistically analysed with Student's t-test or Fisher exact tests. The ELISA results were compared among the three groups by one-way analysis of variance test and Dunnett as post-hoc analysis. Differences in $\mathrm{T}$ cell subpopulation and markers frequencies by flow cytometry were evaluated by multiple t-tests, and a Holm-Sidak was used to adjust for multiple comparisons. Additionally, the correlation age/CD4 and CD8 T cell subpopulations were analysed by straight-line regression, slope and intercept were calculated and compared between elderly with or without ILA. Database, statistics and graphics were made with GraphPad Prism 8 (GraphPad Software, USA).

\section{RESULTS}

\section{Baseline characteristics of study populations}

In this study, we examined 15 subjects with ILA from our Lung Ageing Programme, and 21 age-matched controls randomly selected from the same cohort but with normal HRCT. We also include 28 health younger subjects (age $24 \pm 4$ years old, $54 \%$ males). As expected, individuals with ILA were predominantly smoker males, and we did not find differences in lung function tests (table 1).

According to with the classification proposed by the Fleischner Society, ${ }^{16} 10$ individuals (67\%) showed subpleural fibrotic ILA and 5 individuals (33\%) showed subpleural non-fibrotic ILA (figure 1A,B, respectively).

\begin{tabular}{|c|c|c|c|}
\hline Variable & Old $n=21$ & Old + ILA $n=15$ & $P$ value \\
\hline Age (years) & $67 \pm 6$ & $70 \pm 9$ & 0.2 \\
\hline Gender, male (\%) & $3(14)$ & $12(80)$ & 0.0001 \\
\hline Smoke, former (\%) & $8(38)$ & $12(80)$ & 0.01 \\
\hline FVC (\% predicted) & $104 \pm 33$ & $99.8 \pm 13$ & 0.7 \\
\hline FEV1 (\% predicted) & $103 \pm 20$ & $105 \pm 20$ & 0.7 \\
\hline DLCO (\% predicted) & $113 \pm 21$ & $105 \pm 15$ & 0.2 \\
\hline
\end{tabular}

DLCO, diffusion capacity for carbon monoxide; FEV1, forced expiratory lung volume in the first second; FVC, forced vital capacity; ILA, interstitial lung abnormalities. 

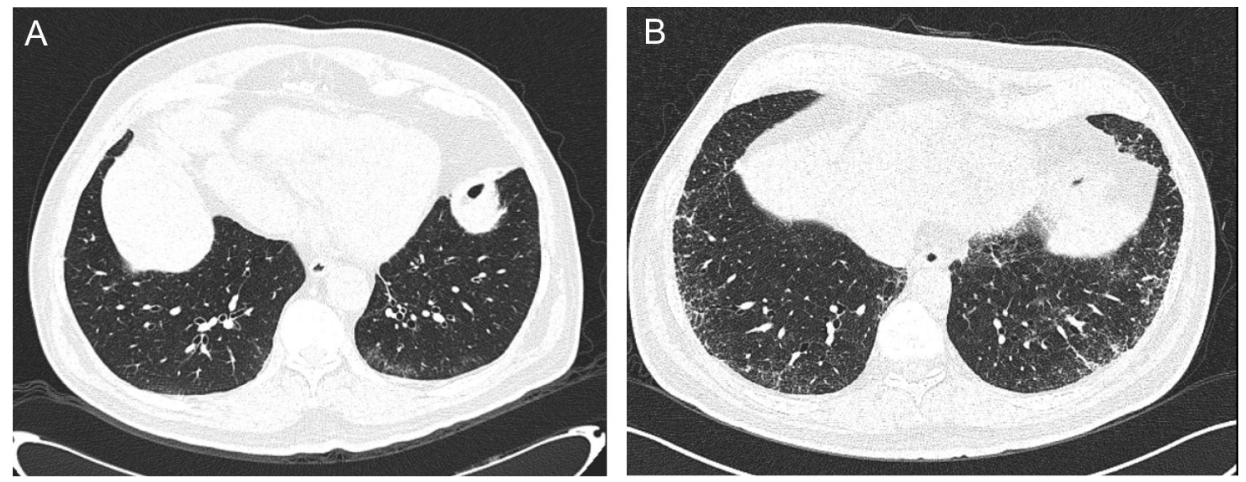

Figure 1 CT scan in two different respiratory asymptomatic subject of our cohort. Panel A: Subpleural non-fibrotic ILA. Panel B: Subpleural fibrotic ILA. ILA, interstitial lung abnormalities.

\section{The distribution of $\mathrm{CD} 4+\mathrm{T}$ cell subpopulations differs} between ILA persons and the normal older control group

Maturity status of $\mathrm{T}$ cells is relevant to induce specific functions, and this status physiologically changes during ageing. ${ }^{12}$ We analysed the frequency of CD4+ and CD8 $+\mathrm{T}$ cells subpopulations by flow cytometry, following the analyses strategy indicated in online supplemental figure S2.

Inside the gate of the CD4+T cell, we first evaluated the frequency of Naïve, $\mathrm{T}_{\mathrm{CM}}, \mathrm{T}_{\mathrm{EM}}$ and TEMRA subpopulations (figure $2 \mathrm{~A}$ ). As expected, the older control group displayed a decreased frequency of
Naïve CD4+T cells with an increment of $\mathrm{T}_{\mathrm{CM}}$ and $\mathrm{T}_{\mathrm{EM}}$ subpopulations compared with the young group, thus presenting the characteristic profile of maturity status subpopulations that occurs during ageing.

In contrast, the ILA group did not follow the same pattern, and the profile of subpopulations distribution was more similar to the younger cohort (figure 2A). Furthermore, in the older control group the loss of Naïve and gain of memory cells correlated directly with their age, demonstrating that the intercept of their lines were significantly different in Naïve, $\mathrm{T}_{\mathrm{CM}}$ and $\mathrm{T}_{\mathrm{EM}} \mathrm{CD} 4+\mathrm{T}$ cells compared with the ILA group (figure 2B).

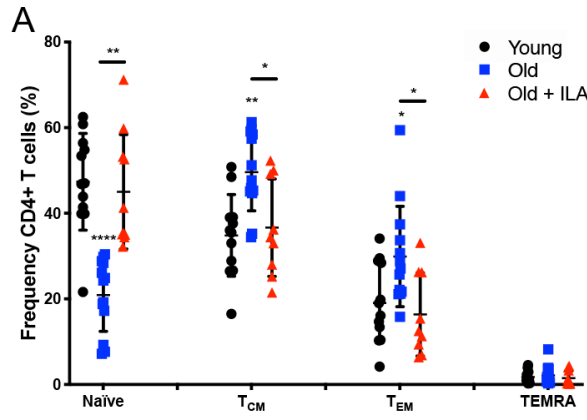

B
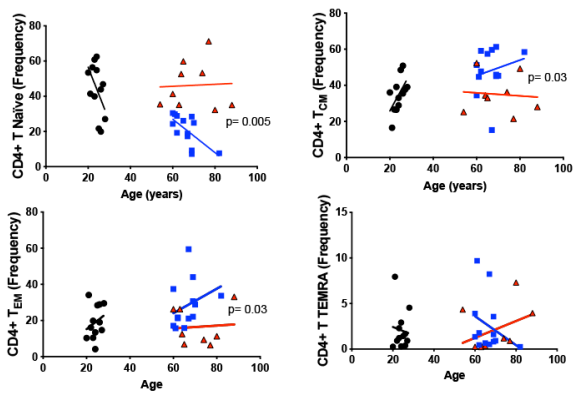

C

D
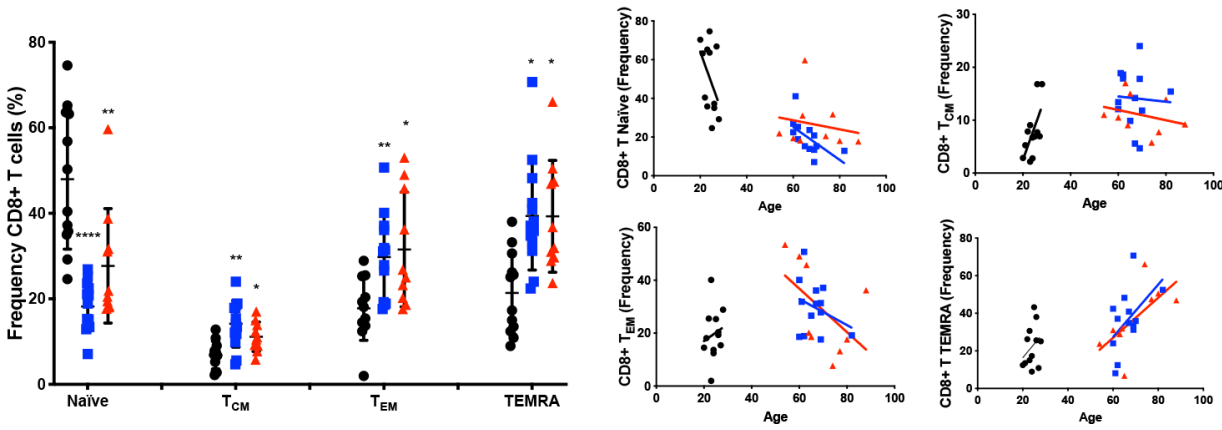

Figure 2 Frequency of naïve and memory CD4+T cells are altered in persons with interstitial lung abnormalities (ILA) compared with old control group. Peripheral mononuclear cells were prepared to flow cytometry. First, live cells were identified, and the gate of $C D 3+C D 4+$ or $C D 3+C D 8+T$ cells was delimited. Then, CCR7 and CD45RA expression were evaluated to obtain the frequency of: Naïve (CD45RA+CCR7+), central Memory (CM, CD45RA-CCR7+), effector Memory (EM, CD45RA-CCR7-) and TEMRA (CD45RA+CCR7-) in CD4+ or CD8+T cells subpopulations (panel A and C, respectively). Panel B: Individual variations in their rate of change of CD4+T cells subpopulations over age; graphic shows the individual-behaviour of each individual, and the regression line per group-age (line colour per group is indicated). Panel D: Individuals vary in their rate of change of CD8+T cells subpopulations over age; graphic shows the individual-behaviour of each individual and the regression line per group-age (line colour per group is indicated). Graphs show individual values, mean \pm SD (young: $n=12$, old: $n=13$, old + ILA: $n=10$ ). ${ }^{*} p<0.05{ }^{* *} p<0.01{ }^{* * *} p<0.0001$. Statistical analysis was performed with multiple t-tests and a Holm-Sidak method was used to adjustment for multiple comparisons; asterisks indicate comparison of the three groups. When the asterisk is on a line, indicates, the comparison between old control group and old +ILA. CCR7,C-C chemokine receptor $7 ; \mathrm{T}_{\mathrm{CM}^{\prime}}$ central memory; $\mathrm{T}_{\mathrm{EM}^{\prime}}$ effector memory; TEMRA, effector memory RA. 
A

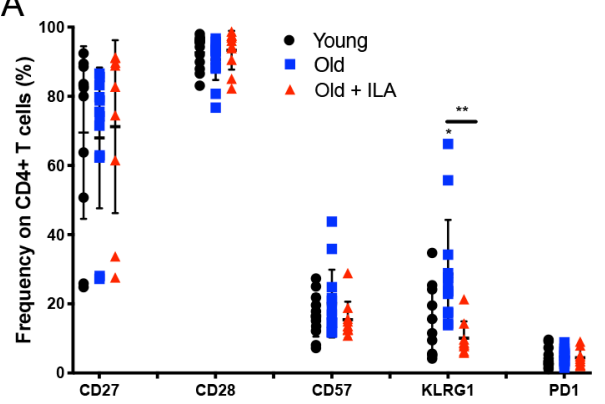

C

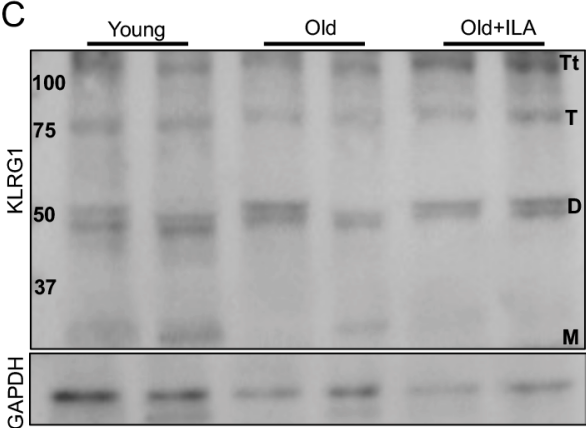

B
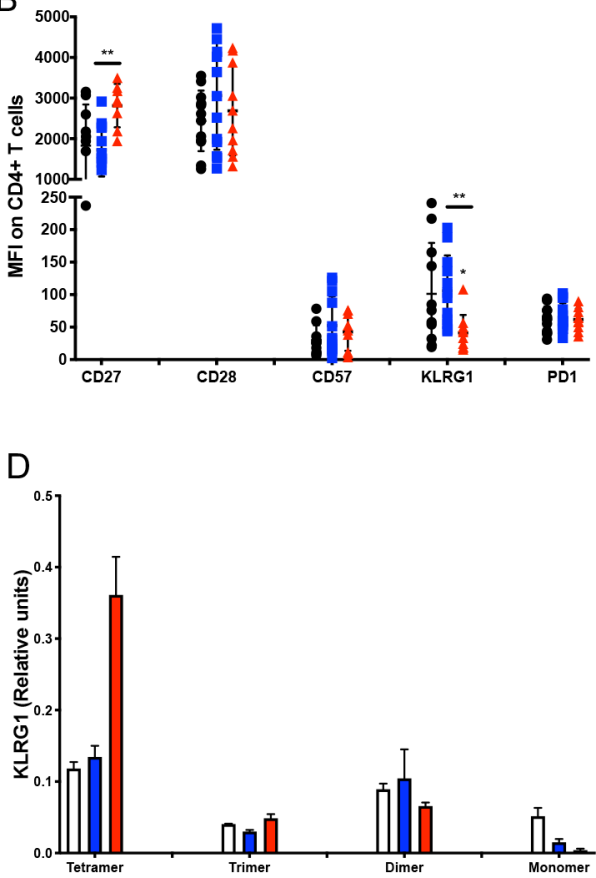

Figure 3 CD4+T cells from subjects with interstitial lung abnormalities (ILA) loss the KLRG1 expression compared with old control group. Peripheral mononuclear cells were prepared to flow cytometry and western blot. First, live cells were identified, the gate of CD3+CD4+was delimited, and then the expression of CD27, CD28, CD57, KLRG1 and PD1 was evaluated by percentage and mean fluorescence intensity (MFI) (panels A and B, respectively). Panel C: Total T cells were obtained using a Pan T kit and then CD4+T cells were enriched. The expression of KLRG1 was evaluated in the fraction of $C D 4+T$ cells by western blot under non-reducing condition. Panel D: Band densities were normalised against GAPDH by densitometry analysis and results are shown in relative units of concentration using IMAGEJ software; bars show mean $\pm S D$. In panels $A$ and $B$, graphs show individual value mean \pm SD from: Young: $n=12$, old: $n=13$, old + ILA: $n=10$. Western blot was performed in two subjects per group. ${ }^{*} p<0.05{ }^{* *} p<0.01$. Statistical analysis was performed with multiple t-tests and a Holm-Sidak method was used to adjustment for multiple comparisons; asterisks indicate comparison of the three groups. When the asterisk is on a line, indicates, the comparison between old control group and old+ILA. CCR7, C-C chemokine receptor 7; KLRG1, killer-cell lectin-like receptor G1; PD-1, programmed cell death 1.

Inside CD8 + T cells, results showed a classic distribution of Naïve, $\mathrm{T}_{\mathrm{CM}}, \mathrm{T}_{\mathrm{EM}}$ and TEMRA during the ageing, characterised by the decrease in the frequency of Naïve and increase of $T_{C M}$, $\mathrm{T}_{\mathrm{EM}}$ and TEMRA without differences between the older control group and ILA cohort (figure 2C). Likewise, CD8+T subsets not correlated with age in both old groups independently of the ILA presence (figure 2D).

\section{CD4+T cells from ILA person loss the high expression of KLRG1 characteristic of ageing}

Next, we evaluated by flow cytometry the expression of markers to differentiate between cellular senescence or exhaustion status. The loss of CD27 and CD28, as well as the high expression of CD57 or KLRG1, is a phenotype associated to cellular senescence, whereas high expression of programmed cell death 1 (PD-1) is associated to exhaustion status. ${ }^{17}$

Thus, CD27, CD28, CD57, KLRG1 and PD-1 expression were evaluated inside $\mathrm{CD} 4+$ and $\mathrm{CD} 8+\mathrm{T}$ cells gates using PBMC as described in online supplemental figure S2. Our results showed that ILA subjects had decreased frequency of KLRG1+CD4+T cells compared with the older control group, while the expression of the other molecules was not affected (figure 3A). Interestingly, KLRG1 was also decreased in the intensity of expression as compared with the older control group (figure 3B). Also, CD4+T cells from ILA persons have increased intensity of CD27 expression.

KLRG1 is an inhibitory receptor on $\mathrm{T}$ cells, and it has been suggested that the multimerisation of KLRG1 increases the avidity to its ligand E-cadherin, and consequently enhance the sensitivity to induce the inhibitory activity on T cells. ${ }^{15}$ We purified CD4+and CD4 - T cells from our study groups to evaluate the KLRG1 isoforms by western blot (figure 3C). Our data suggest that CD4+T cells from ILA persons have predominantly the $\mathrm{Tt}$ isoform with loss of the $\mathrm{M}$ isoform, probably as a compensatory mechanism to the low frequency of KLRG1+CD4+T cells (figure 3D).

Contrary, CD8+T cells from ILA persons display a similar profile of senescence and exhaustion status than the older control group. As is illustrated in online supplemental figure S4A,B, $\mathrm{CD} 8+\mathrm{T}$ cells phenotype in both groups were characterised by the decrease of frequency and mean fluorescence intensity of CD27 and CD28, and the increase of CD57. KLRG1 expression was not affected, and isoforms were not detected by western blot (online supplemental figure S4C).

\section{CD4+T cells from ILA are highly proliferative}

In order to clarify the activation status of $\mathrm{CD} 4+\mathrm{T}$ cells from ILA persons, we purified T cells and stimulated them with anti-CD3/ anti-CD28 or phorbol myristate acetate (PMA)/ionomycin to verify their activation and proliferation ability. Results of T-cells from the younger group were considered as a reference value, normalised to $100 \%$.

CD25 expression was evaluated by flow cytometry as indicative of activation status. We were not able to identify a statistical difference in the expression of this marker. However, the older control group had $50 \%$ more frequency of $\mathrm{CD} 25+\mathrm{CD} 4+\mathrm{T}$ 

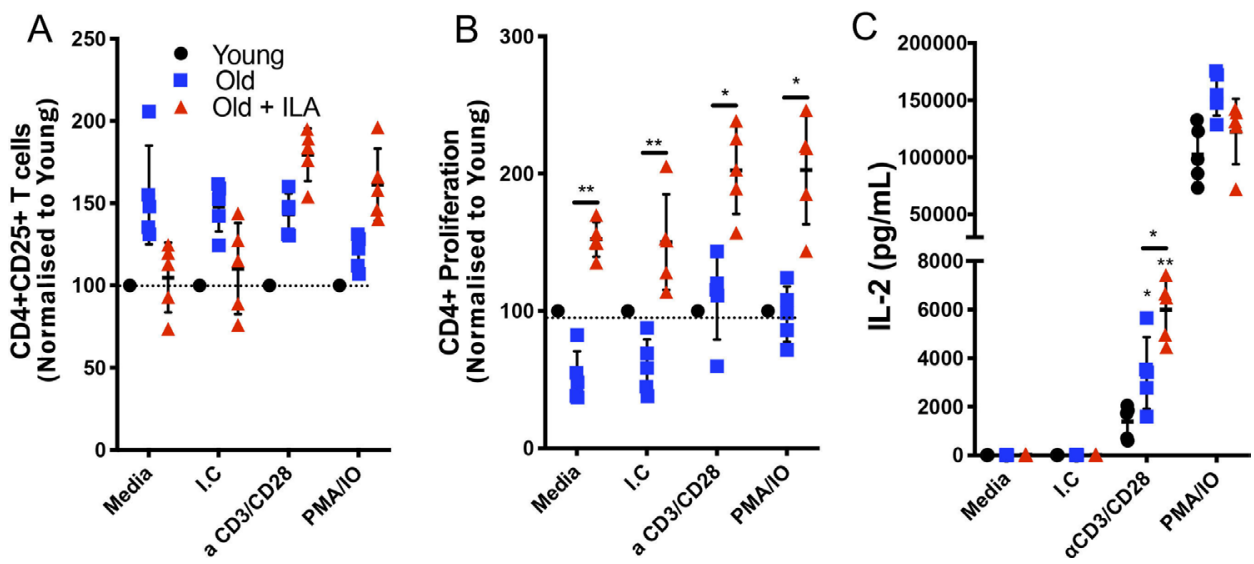

Figure 4 CD4 +T cells from persons with interstitial lung abnormalities (ILA) display high proliferative capacity compared with old control group. Enriched T cells were cultured 48 hours with polyclonal stimuli using either monoclonal antibodies anti-CD3 and CD28 (both at $1 \mu \mathrm{gg} / \mathrm{mL}$ ) or phorbol myristate acetate (PMA) $50 \mathrm{nM}$ /ionomycin (IO) $2 \mathrm{ng} / \mu \mathrm{L}$, culture medium (Media) and isotype control (I.C) to monoclonal antibodies were used as negative controls. Cells were recovered and prepared to flow cytometry and culture supernatants were used to determine interleukin (IL)-2 by ELISA. By flow cytometry, CD3+CD4+cells were delimited and the expression of CD25 (for activation) and Ki-67 (for proliferation) were evaluated (panel A and $B$, respectively); the level of activation and proliferation displayed for CD4+T cells from young group was considered as $100 \%$ (black dot line). Panel C: IL-2 released by stimulated T cells was measured by ELISA in the culture' supernatants. Graphs show individual value mean \pm SD $(n=5$ per group). ${ }^{*} p<0.05{ }^{*} p<0.01$. Statistical analysis was performed with one-way analysis of variance test and Dunnett as post hoc analysis (ELISA data), and multiple t-tests with a Holm-Sidak to adjustment for multiple comparisons (flow cytometry data). Asterisks indicate comparison of the three groups. When the asterisk is on a line, indicates, the comparison between old control group and old+ILA. M-CSF, macrophage colony-stimulating factor; TNF, tumor necrosis factor.

cells than the young group, and this profile was maintained at basal conditions and after any of the stimulation conditions (figure 4A). By contrast, under basal conditions, the frequency of CD25+CD4+T cells in the ILA group was similar to the younger group and also increased under stimulation (figure 4A). Importantly, in comparison with the older controls, CD4+T cells from ILA group, exhibited a significantly higher proliferative background from the basal condition that was maintained under both stimulation conditions (figure 4B).

Finally, we measured the levels of IL-2 in culture supernatant as an indirect marker of activation and proliferation status. As we expected, CD4+T cells from the old control cohort increased the IL-2 level in response to anti-CD3/anti-CD28 stimuli, but under the same condition, ILA group released near double levels of IL-2 (figure 4C). However, under PMA/ionomycin stimuli both old controls and ILA subjects produced high and similar levels of IL-2 (figure 4C). Therefore, CD4+T cells from ILA are highly activated under specific anti-CD3/anti-CD28 stimulus where KLRG1 should inhibit it, but not when treated with PMA/ ionomycin because this stimulus induces activation down-stream of KLRG1 inhibition. Together, these data suggest that CD4+T cells from ILA group are more sensitive to activation and proliferation likely associated with the loss of KLRG1 expression.

Regarding activation and proliferation status of CD8+T cells, we did not observe differences between older control and ILA groups (online supplemental figure S5A,B). However, a decreased level of granzyme B under both stimulation conditions was observed in culture supernatants from the ILA group (online supplemental figure S5C).

\section{CD4+T cells from ILA subjects secrete a different cytokine profile compared with age-matched normal controls}

$\mathrm{CD} 4+\mathrm{T}$ cells mediate the immune response through the secretion of specific cytokines; a simplified form to divide the function of the cytokine families is according their effects as proinflammatory and anti-inflammatory subsets, although in recent years, subsets with more specialised and defined properties were described. ${ }^{18}$

Because CD4+T cells from ILA group have loss KLRG1 expression and they have a high background of proliferation and activation, we performed a wide screening of cytokines/chemokines released in the culture supernatants to clarify whether CD $4+\mathrm{T}$ cells from ILA group has a specific profile. From a panel of 48 molecules, we observed a different profile between older control and ILA groups in the release of 10 cytokines, 3 growthfactors and 1 chemokine (figure 5).

From them, IL- 6 and IFN- $\gamma$ levels were increased at the basal condition in ILA's CD4+T cells, but in response to the stimulus, both study groups produced similar amounts of these cytokines (figure 5A). These results demonstrated by the first time that $\mathrm{T}$ cells from ILA group are continually delivering high levels of IL- 6 and IFN- $\gamma$. Interestingly, the increase of the different cytokine/chemokine levels was dependent of the stimulus received; IL1- $\beta$ and IL- 8 were increased after both anti$\mathrm{CD} 3 / \mathrm{CD} 28$ and PMA/IO (figure $5 \mathrm{~B}$, up-line), suggesting that the synthesis of IL-1 $\beta$ and IL- 8 could be induced independently of the alteration in the KLRG1 pathway. However, IL-18 and the chemokine CXCL10 were increased specifically under antiCD3/CD28 stimuli (figure 5B, middle-line), whereas IL-15, G-CSF and VEGF levels were increased only under PMA/IO stimuli (figure $5 \mathrm{~B}$, low-line). $\mathrm{T}$ cells from ILA group delivered lower lymphotoxin levels than the older control group under both anti-CD3/CD28 and PMA/IO stimulus, but IL-17 and macrophage colony- stimulating factor (M-CSF) levels were decreased only under anti-CD3/CD28 stimuli (figure 5C, up-line) while the tumor necrosis factor (TNF) and IL-12p70 levels were decreased only under PMA/IO stimuli (figure 5C, low-line).

The other analysed proteins did not show differences (online supplemental figure S6). Some proteins levels were out of the limit detection; for example, IL-2 and MIP-1a levels were up limit whereas IL-5 was down limit (data not shown). 
A
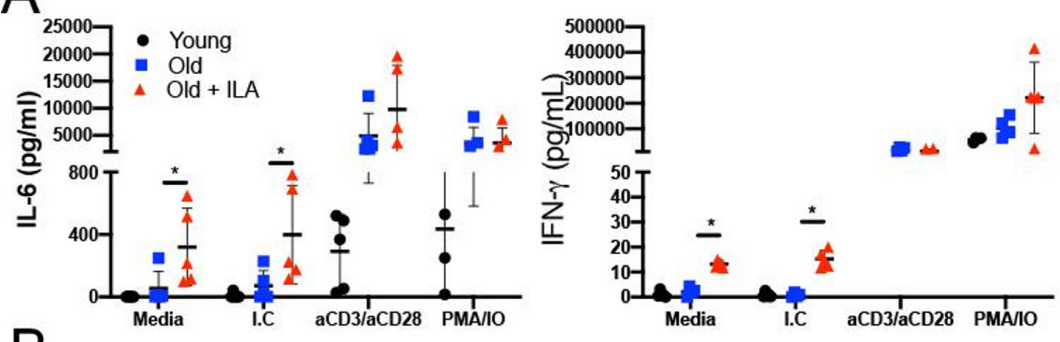

B
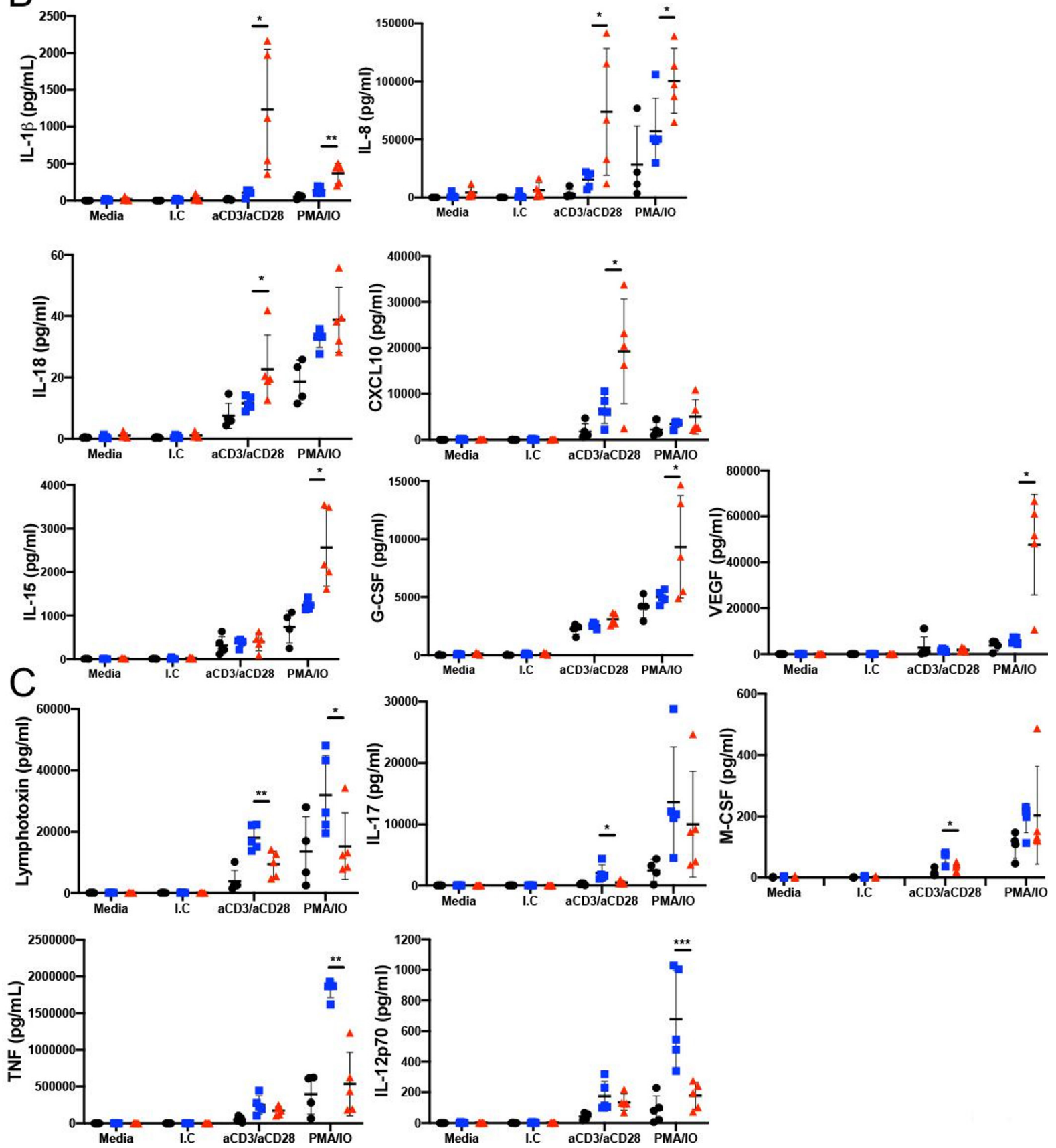

Figure 5 Under polyclonal stimuli the T cells from persons with interstitial lung abnormalities (ILA) secrete a specific pro-inflammatory profile. Enriched T cells were cultured 48 hours with polyclonal stimuli either with monoclonal antibodies anti-CD3 and CD28 (both at $1 \mu \mathrm{g} / \mathrm{mL}$ ) or phorbol myristate acetate (PMA) $50 \mathrm{nM} /$ /ionomycin (IO) $2 \mathrm{ng} / \mu \mathrm{L}$; culture medium (Media) and isotype control (I.C) to monoclonal antibodies were used as negative controls. Culture supernatant was used to evaluate several molecules by Multiplex. We observed diverse behaviour in the profile of delivered pro-inflammatory cytokines; a first cytokine group was spontaneously release (stimuli-independent), a second group showed increased level and a third group showed decreased level in response to polyclonal stimuli (panel A, B and C, respectively). Graphs show individual value mean $\pm S D(n=5$ per group). ${ }^{*} p<0.05{ }^{* *} p<0.01{ }^{* * *} p<0.001$ compared old versus old+ILA, statistical analysis was performed with one-way analysis of variance tests and adjusted by the Holm-Sidak method for multiple comparisons. IFN- $\gamma$, interferon gamma; IL, interleukin. 
A
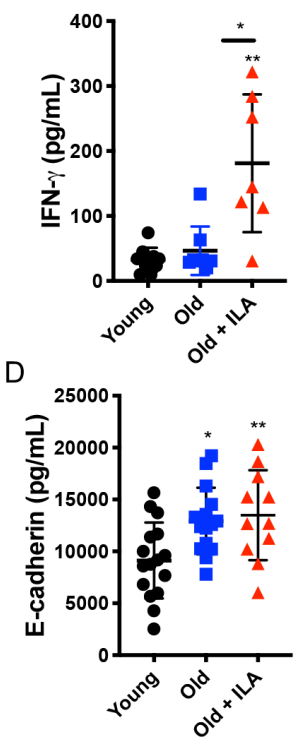

B

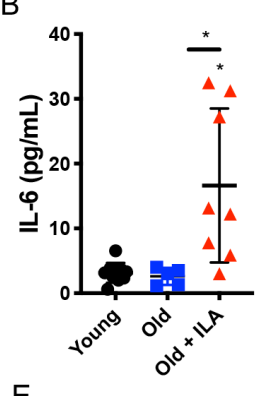

C

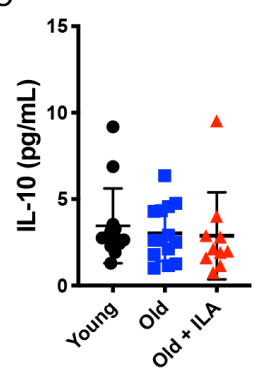

$\mathrm{E}$

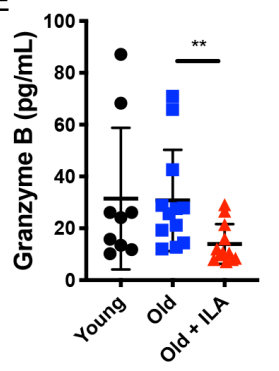

Figure 6 Individuals with interstitial lung abnormalities (ILA) display high levels of pro-inflammatory cytokines in plasma. Plasma was obtained and prepared to measure several soluble proteins by ELISA. Plasma concentrations of IFN- $\gamma$, IL-6, IL-10, E-cadherin and granzyme $B$ were evaluated (panel $A, B, C, D$ and E, respectively). Graphs show individual value mean $\pm S D$ (young: $n=16$, old: $n=15$, old + ILA: $n=11$ ). ${ }^{*} p<0.05{ }^{* *} p<0.01$. Statistical analysis was performed with an one-way analysis of variance test adjusted by the Dunnett method; asterisks indicate comparison of the three groups. When the asterisk is on a line, indicates, the comparison between old control group and old+ILA. IFN$\gamma$,interferon gamma; IL, interleukin.

Together, our data suggest that $\mathrm{T}$ cells from ILA persons are able to secrete a specific pro-inflammatory profile, characterised by high production of cytokines such as IFN- $\gamma$, IL-6, IL-1 $\beta$, IL-8 and IL-18. Nevertheless, interestingly, despite this pro-inflammatory profile, they produce lower classical proinflammatory cytokines such as TNF or lymphotoxin.

\section{Concentrations of IFN- $\gamma$ and IL- 6 are increased in plasma from ILA subjects}

Since we found in vitro that T cells from ILA group induced a pro-inflammatory cytokine profile, we hypothesised that ILA persons might have high levels of some pro-inflammatory cytokines in the blood. In order to confirm our hypothesis, we used an ELISA assay and measured the plasma levels of IFN- $\gamma$ and IL-6 as pro-inflammatory cytokines as well as IL-10 as an antiinflammatory cytokine.

Our data showed that ILA subjects have significantly higher levels of IFN- $\gamma$ and IL- 6 compared with both the younger and older control groups (figure 6A,B, respectively), whereas, IL-10 level did not show differences between groups (figure 6C). In addition, the levels of soluble E-cadherin were increased in both older controls and ILA group compared with the young cohort (figure 6D). Interestingly, soluble granzyme B, an effector molecule produced by $\mathrm{CD} 8+\mathrm{T}$ cells, was decreased in plasma from ILA group compared with older control cohort (figure 6E), paralleling our findings when CD8+T cells were stimulated in vitro (online supplemental figure 55C). Together, these results suggest that ILA subjects have a systemic pro-inflammatory profile likely associated with the activation of CD4+T cells, and that probably the cytotoxic function of $\mathrm{CD} 8+$ is affected.

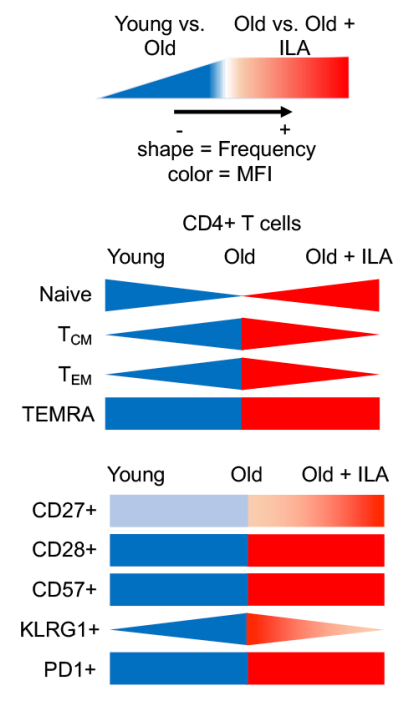

Figure 7 Summary of main differences in the phenotype of $C D 4+T$ cells comparing young-old-old/interstitial lung abnormalities (ILA). The decrease of the frequency of naïve and the increase of memory CD4+T cells, as well as the loss of the co-stimulatory molecule CD27 and the gain of the 'brake molecule' KLRG1 is considered a normal process in ageing, as illustrated when compare young versus old persons. By contrast, our data showed that old//LA does not show this typical phenotype profile, when compared with old controls; actually, old/LA is characterised by increased frequency of naïve and decreased frequency of memory CD4+T cells, and these cells do not lose the expression of CD27 and do not express KLRG1. KLRG1, killer-cell lectin-like receptor G1; MFI, mean fluorescence intensity; PD-1, programmed cell death 1; $\mathrm{T}_{\mathrm{CM}^{\prime}}$, entral memory; $\mathrm{T}_{\mathrm{EM}^{\prime}}$ effector memory; TEMRA, effector memory RA.

\section{DISCUSSION}

Interstitial lung abnormalities are observed in a small but significant percentage of older, mostly smoker individuals. Longitudinal follow-up studies have demonstrated that ILA persists in about $60 \%$ of affected individuals and more important, that progress in $20 \%$ to $40 \%$ within 6 years and is associated with a higher risk of all-cause mortality. ${ }^{79} 19$

It is well known that with age there is a progressive decline in the capacity of mounting a robust $\mathrm{T}$ cells response, among other reasons, by the loss of Naïve $\mathrm{T}$ cells and accumulation of memory T cells. ${ }^{11}{ }^{12}$ Our findings indicate that ILA persons exhibit a different immunological profile compared to normal old individuals with similar demographic characteristics and that these differences affect primarily $\mathrm{CD} 4+\mathrm{T}$ cells both in phenotype and function.

ILA group displayed an imbalance in $\mathrm{CD} 4+\mathrm{T}$ cells subpopulations, characterised by an increase in the frequency of Naïve $\mathrm{T}$ cells, and a decrease of memory subsets. Probably the high frequency of naïve CD4+T cells is favouring the recognising of a vast repertory of potential new antigens. Additionally, CD4+T cells subpopulations from ILA subjects compared with the old control group showed higher intensity of CD27 expression, and they did not gain the expression of the proliferative-limiting, co-inhibitory receptor KLRG1, which characteristically occurs in the normal ageing process (figure 7). Moreover, our data showed that these cells had increased proliferative and functional activities at least in part associated with the decreased frequency of KLRG1+CD4+T cells.

Even if CD4+T cells from ILA expressed the Tt form of KLRG1, perhaps as a compensatory mechanism to its low 

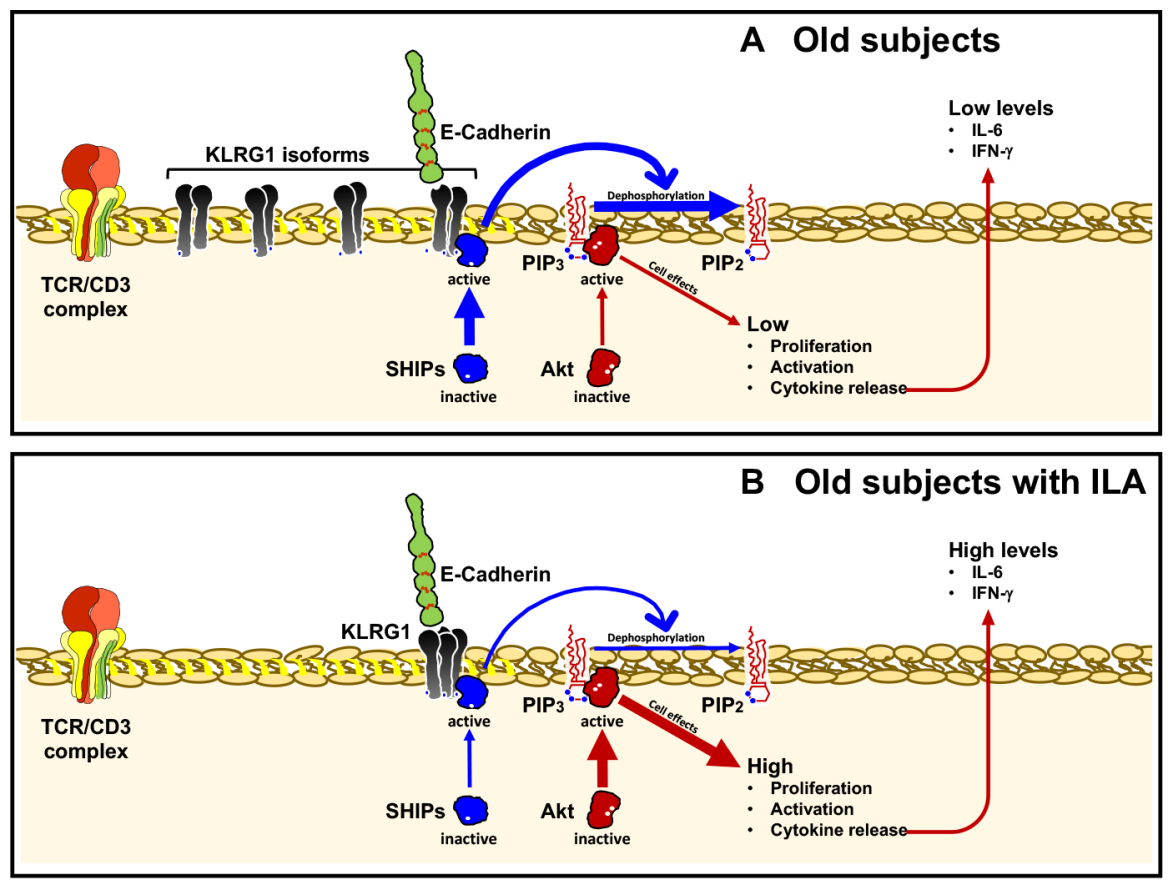

Figure 8 Schematic summary of the consequences of lack of KLRG1 expression on CD4+T cells from old+ILA patients. Panel A: KLRG1 is overexpressed on CD4+T cells in old subjects. After KLRG1 ligation, its inhibitory effect occurs through the recruitment of SHIP-1 and SHIP-2 phosphatases, which negatively regulate the function of PI3K and consequently the serine-threonine kinase Akt is inhibited, as well as the TCR signalling. Panel B: CD4+T cells from ILA are deficient in KLRG1 expression and consequently the inhibitory signalling is not activated. Then, CD4+T cells display an enhanced activation status and a pro-inflammatory functional profile, which is characterised by the high production of cytokines like IL-6 and IFN- $\gamma$.

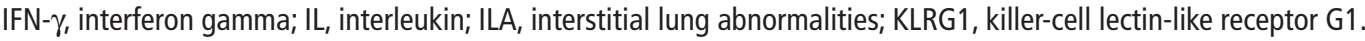

expression, this was not enough to avoid that CD4+T cells from ILA group were highly proliferative. Typically, KLRG1 exerts its inhibitory effects through one ITIM motif contained in its cytoplasmic domain and recruiting SHIP-1 and SHIP-2 phosphatases, which negatively regulate the function of PI3K. This pathway inhibits the activation of the serine-threonine kinase Akt affecting the activation of several kinases, transcription factors and other regulatory molecules. ${ }^{20}$ (figure 8A).

The loss of KLRG1 expression on CD4+T cell from ILA is perpetuating an unspecific activation, as demonstrated by our data which show that even without stimuli these cells present twofold proliferation compared with CD4+T cells from older control group which, as expected, displayed increased KLRG1 expression and low proliferation compared with younger group (figure 8B).

Additionally, CD4+T cells from ILA group release a characteristic pro-inflammatory profile, either spontaneously or after stimulation. In culture, these cells delivered spontaneously high levels of IL- 6 and IFN- $\gamma$, which resulted also increased at the systemic level (in plasma). These findings suggest that IL- 6 and IFN- $\gamma$ could be biomarkers for early identification of ILA, which is relevant because currently, the identification of this disorder rests only on HRCT.

Our results are in concordance with observations in other models, where the lack of KLRG1 expression on T-cells conferred them a functional competitive advantage, because the KLRG1 ligation decreases the TCR signalling, inhibiting the proliferative capacity and decreasing cytokine production. ${ }^{21} 22$ We hypothesised that the high production of cytokines, like IL- 6 and IFN- $\gamma$, and proliferative ability of CD4+T cells in ILA subjects might be independent of their differentiation status (naïve vs memory). However, probably it is a result of the lack of KLRG1 expression, which is a 'brake-molecule'.
Moreover, under polyclonal stimuli, CD4+T cells from ILA persons released significant amounts of a variety of proinflammatory cytokines, mainly those related to the inflammasome such as IL-1 $\beta$, IL- 8 and IL-18. IL-1 $\beta$ and IL-18 are members of the IL- 1 family and are critical mediators of inflammation in several pathologies, including infectious and autoimmune disorders. ${ }^{23}$ Signalling through IL-18 induces transcription and secretion of a subset of pro-inflammatory molecules, including IFN- $\gamma$ by CD4+T cells as we observed in our study. ${ }^{24}$ Whereas IL-8, is a chemokine essential for neutrophilmediated inflammation and angiogenesis.

Curiously, also under polyclonal stimuli, CD4+T cells from ILA persons, compared with older, lost their ability to produce the classical pro-inflammatory cytokines such as TNF and IL-17. This profile was similar to younger controls, suggesting that both ILA and young groups still conserve other pathways to control an excessive secretion of specific cytokines like TNF and IL-17.

Together, these data indicate that the deregulated cytokine/ chemokine profile displayed by CD4+T cells from ILA persons may contribute to a higher systemic pro-inflammatory status and the development of the interstitial pulmonary abnormalities.

In sharp contrast, no differences between the ILA and the older control groups were observed in the distribution of CD8+ subpopulations, as well as in the expression of immunosenescence markers and the proliferative capacity. However, both systemic and in vitro levels of granzyme B were decreased in ILA persons. Probably, its decreased expression may be a compensatory anti-apoptotic mechanism since, for example, reduced granzyme B expression in different mouse models is associated with improved wound healing and less inflammation and fibrosis compared with control mice. ${ }^{25}$ 
Our results of CD8 + T cells in ILA open several questions; we did not clarify if the function of these cells is affected. Unfortunately, studies on phenotype and function of CD8 $+\mathrm{T}$ cells in ageing and immunosenescence are limited, ${ }^{26} 27$ and further research is necessary to identify the contribution of CD8+T cells in the development of ILA.

Our study has limitations, such as the small sample size and the heterogeneity of the individuals with ILA included in the study (subpleural fibrotic and subpleural non-fibrotic). However, our findings are consistent to indicate, by the first time, that CD4+T-lymphocytes from ILA subjects display high proliferative activity, likely related to the loss of KLRG1 expression, and a proinflammatory profile, which may contribute to the development of ILA.

\section{Twitter Leslie Chavez-Galan @ChavezGalan78}

Acknowledgements The authors are grateful to the patients of the Lung Ageing Programme for their generous collaboration.

Contributors LC-G and MS conceived and designed the study. CM, RO-G and LC-G performed the experiments. CM, IB-R, LC-G, MM-M, AP and MS examined and interpreted the experimental data. IB-R and MM-M recruited patients and analysed clinical data. CM, IB-R, MM-M and AP contributed to the manuscript preparation. LC-G wrote the manuscript with input from all co-authors. LC-G and MS oversaw all experimental design and manuscript preparation.

Funding This study was supported by Secretaría de Educación, Ciencia, Tecnología e Innovación de la Ciudad de Mexico, grant: SECITI/115/2017 awarded to IB-R; and by CONACYT, grant: FOSISS 290645 awarded to IB-R.

Competing interests None declared.

Patient consent for publication Obtained.

Ethics approval Ethics committee/institutional review boards approved the study, protocol numbers: C39-14 and B16-19.

Provenance and peer review Not commissioned; externally peer reviewed.

Data availability statement All data relevant to the study are included in the article or uploaded as supplementary information. Authors confirm that the raw data to support the conclusions of this study are included in the manuscript. Corresponding author will provide more information, upon rational request, to any qualified researcher.

Open access This is an open access article distributed in accordance with the Creative Commons Attribution Non Commercial (CC BY-NC 4.0) license, which permits others to distribute, remix, adapt, build upon this work non-commercially, and license their derivative works on different terms, provided the original work is properly cited, appropriate credit is given, any changes made indicated, and the use is non-commercial. See: http://creativecommons.org/licenses/by-nc/4.0/.

\section{ORCID iDs}

Carlos Machahua http://orcid.org/0000-0003-4964-7393

Ivette Buendia-Roldan http://orcid.org/0000-0002-2825-506X

Ranferi Ocaña-Guzman http://orcid.org/0000-0002-9884-7386

Annie Pardo http://orcid.org/0000-0003-2168-9073

Leslie Chavez-Galan http://orcid.org/0000-0002-2334-0361

Moises Selman http://orcid.org/0000-0002-1022-4783

\section{REFERENCES}

1 Thannickal VJ, Murthy M, Balch WE, et al. Blue journal conference. aging and susceptibility to lung disease. Am J Respir Crit Care Med 2015;191:261-9.
2 Budinger GRS, Kohanski RA, Gan W, et al. The intersection of aging biology and the pathobiology of lung diseases: a joint NHLBI/NIA workshop. J Gerontol A Biol Sci Med Sci 2017;72:1492-500.

3 Faner R, Rojas M, Macnee W, et al. Abnormal lung aging in chronic obstructive pulmonary disease and idiopathic pulmonary fibrosis. Am I Respir Crit Care Med 2012;186:306-13.

4 Washko GR, Hunninghake GM, Fernandez IE, et al. Lung volumes and emphysema in smokers with interstitial lung abnormalities. N Eng/ J Med 2011;364:897-906.

5 Hunninghake GM, Hatabu H, Okajima Y, et al. MUC5B promoter polymorphism and interstitial lung abnormalities. N Engl J Med 2013;368:2192-200.

6 Podolanczuk AJ, Oelsner EC, Barr RG, et al. High attenuation areas on chest computed tomography in community-dwelling adults: the MESA study. Eur Respir J 2016;48:1442-52.

7 Araki T, Putman RK, Hatabu H, et al. Development and progression of interstitial lung abnormalities in the Framingham heart study. Am J Respir Crit Care Med 2016;194:1514-22.

8 Buendia-Roldan I, Machuca N, Mejía M, et al. Lower levels of $\alpha$-Klotho in serum are associated with decreased lung function in individuals with interstitial lung abnormalities. Sci Rep 2019;9:10801.

9 Putman RK, Hatabu H, Araki T, et al. Association between interstitial lung abnormalities and all-cause mortality. JAMA 2016;315:672-81.

10 Förster R, Davalos-Misslitz AC, Rot A. CCR7 and its ligands: balancing immunity and tolerance. Nat Rev Immunol 2008;8:362-71.

11 Linton PJ, Dorshkind K. Age-Related changes in lymphocyte development and function. Nat Immuno/ 2004;5:133-9.

12 Larbi A, Fulop T. From "truly naïve" to "exhausted senescent" T cells: When markers predict functionality. Cytometry Part A 2014;85:25-35.

13 Gründemann C, Bauer M, Schweier O, et al. Cutting edge: identification of Ecadherin as a ligand for the murine killer cell lectin-like receptor G1.J Immunol 2006;176:1311-5.

14 Voehringer D, Koschella M, Pircher H. Lack of proliferative capacity of human effector and memory T cells expressing killer cell lectinlike receptor G1 (KLRG1). Blood 2002;100:3698-702.

15 Rosshart S, Hofmann M, Schweier O, et al. Interaction of KLRG1 with E-cadherin: new functional and structural insights. Eur I Immunol 2008;38:3354-64.

16 Hatabu H, Hunninghake GM, Richeldi L, et al. Interstitial lung abnormalities detected incidentally on CT: a position paper from the Fleischner Society. Lancet Respir Med 2020;8:726-37.

17 Xu W, Larbi A. Markers of T cell senescence in humans. Int J Mol Sci 2017;18:1742.

18 Luckheeram RV, Zhou R, Verma AD, et al. CD4 ${ }^{+}$T cells: differentiation and functions. Clin Dev Immunol 2012;2012:925135.

19 Jin GY, Lynch D, Chawla A, et al. Interstitial lung abnormalities in a CT lung cancer screening population: prevalence and progression rate. Radiology 2013;268:563-71.

20 Henson SM, Akbar AN. KLRG1--more than a marker for T cell senescence. Age 2009;31:285-91.

21 Meinicke $\mathrm{H}$, Bremser A, Brack M, et al. Klrg1 impairs regulatory T-cell competitive fitness in the gut. Immunology 2017;152:65-73.

22 Li L, Wan S, Tao K, et al. Klrg1 restricts memory T cell antitumor immunity. Oncotarget 2016;7:61670-8

23 Dinarello CA. Interleukin-1 in the pathogenesis and treatment of inflammatory diseases. Blood 2011;117:3720-32.

24 Novick D, Kim SH, Fantuzzi G, et al. Interleukin-18 binding protein: a novel modulator of the Th1 cytokine response. Immunity 1999;10:127-36.

25 Turner CT, Hiroyasu S, Granville DJ. Granzyme B as a therapeutic target for wound healing. Expert Opin Ther Targets 2019;23:745-54.

26 Briceño 0 , Lissina $A$, Wanke $K$, et al. Reduced naïve CD8(+) T-cell priming efficacy in elderly adults. Aging Cell 2016;15:14-21.

27 Song Y, Wang B, Song R, et al. T-cell Immunoglobulin and ITIM Domain Contributes to CD8 ${ }^{+}$T-cell Immunosenescence. Aging Cell 2018;17:e12716. 